\title{
Promotive Effects of Yokukansan, a Traditional Japanese Medicine, on Proliferation and Differentiation of Cultured Mouse Cortical Oligodendrocytes
}

\author{
Toshiyuki Ueki*, Yasushi Ikarashi, Zenji Kawakami, Kazushige Mizoguchi, Yoshio Kase \\ Tsumura Research Laboratories, Kampo Scientific Strategies Division, Tsumura \& Co., Ami-machi, Japan \\ Email: ueki toshiyuki@mail.tsumura.co.jp
}

Received 14 April 2014; revised 29 May 2014; accepted 11 June 2014

Copyright (C) 2014 by authors and Scientific Research Publishing Inc. This work is licensed under the Creative Commons Attribution International License (CC BY). http://creativecommons.org/licenses/by/4.0/

(c) (i) Open Access

\begin{abstract}
Effects of yokukansan, a traditional Japanese medicine, on proliferation and differentiation of oligodendrocytes were examined using purified mouse cortical oligodendrocyte precursor cells (OPCs). OPCs were cultured for four days, and proliferation was evaluated by counting A2B5 (a specific antibody to OPC)-reactive cells on the second day of cell culture. Differentiation from OPC to oligodendrocyte was evaluated by counting 04 (a specific antibody to detect differentiated cells in various stages)-reactive cells on the fourth day of culture. The effects of yokukansan (final concentration: 100 or $200 \mu \mathrm{g} / \mathrm{ml}$ ) on proliferation and differentiation were examined by adding it to the medium for four days. Yokukansan increased not only the number of A2B5-positive cells on the second day but also the number of 04-positive cells on the fourth day compared to those in the corresponding controls. A WST-8 assay was used to identify active components from seven components of Uncaria Hook (UH), one of the constituent galenicals of yokukansan. Geissoschizine methyl ether (GM: $0.1-3.0 \mu \mathrm{M}$ ) was identified by this screening assay and increased the number of A2B5-positive cells on the second day and 04-positive cells on the fourth day as yokukansan did. These results suggest that yokukansan promotes the proliferation and differentiation of oligodendrocytes, and also that GM contained in UH is one of active components responsible for this effect of yokukansan.
\end{abstract}

\section{Keywords}

Yokukansan, Oligodendrocyte, Proliferation, Differentiation, Geissoschizine Methyl Ether

\footnotetext{
${ }^{*}$ Corresponding author.
} 


\section{Introduction}

Oligodendrocytes constitute the myelin sheath along the course of an axon in the central nervous system (CNS). The cytoplasm of the oligodendrocyte is characterized by rough endoplasmic reticulum, but its most prominent characteristic is enclosure of the axon by concentric layers of its own surface membrane. These concentric layers grow so closely together that the oligodendrocyte cytopwlasm is completely extruded and the original internal surfaces of the membrane become fused, producing the ring-like appearance of the myelin sheath in cross section. The myelin sheath wrapping axons in a concentric manner facilitates the rapid saltatory conduction of electrical impulses along the axon [1] [2], which contributes to various neuronal functions including physiological neuropsychiatric functions in the CNS. Therefore, any abnormality of the myelin sheath, such as demyelination, induces neuronal dysfunction. In many cases, demyelination is followed by remyelination, a spontaneous repair process in which new myelin sheaths are elaborated. Remyelination in the CNS is mediated by a multipotent adult stem/precursor cell population traditionally referred to as oligodendrocyte precursor cells (OPCs) [3]. However, in patients with multiple sclerosis (MS), this recovery process ultimately fails, and persistent demyelination with the subsequent axonal loss results in the progression of irreversible functional deficits [1] [4]. A similar demyelination is reported in the aged rat brain [5], hypoxia [6], and thiamine-deficient Wernicke's encephalopathy [7]. Restoration of the signaling pathways for oligodendrocyte differentiation and/or myelination is emerging as a novel and potentially important therapeutic strategy for demyelinating diseases [4] [8] [9].

Yokukansan is one of the traditional Japanese medicines called "kampo" medicines in Japan. It is composed of seven kinds of dried medical herbs. Yokukansan has been approved by the Ministry of Health, Labor, and Welfare of Japan as a remedy for neurosis, insomnia, and irritability in children. Recently, yokukansan was reported to improve behavioral and psychological symptoms of dementia (BPSD), such as hallucinations, agitation, and aggressiveness in patients with Alzheimer's disease, dementia with Lewy bodies, vascular dementia, and other forms of senile dementia [10]-[14]. Recently, we examined the effects of yokukansan on memory and BPSD-like behavior in thiamine-deficient (TD) rats, and demonstrated that it ameliorated not only the behavioral and psychological symptoms [15] and degeneration of oligodendrocytes [16] but also removed the myelin debris (or demyelination) observed in TD rats. These results suggested that the effectiveness of yokukansan may be related to the function or repair of oligodendrocytes, which are responsible for the formation of myelin sheaths.

The first aim of the present study, therefore, was to clarify whether or not yokukansan affects the development of oligodendrocytes. To clarify this issue, the effects of yokukansan on proliferation and differentiation were examined using purified mouse cortical OPCs. The second aim was to clarify the active components in yokukansan. Yokukansan is composed of seven dried medicinal herbs. Among them, Uncaria Hook (UH) has been reported to be one of the active galenicals responsible for the psychotropic effects of yokukansan [17] [18]. In the present study, thus, we focused on UH to find active components that affect the proliferation and differentiation of oligodendrocytes.

\section{Materials and Methods}

\subsection{Test Substance}

The yokukansan used in the present study was a dry powdered extract [19] from a mixture of Atractylodes Lancea Rhizome (4.0 g, rhizome of Atractylodes Lancea De Candolle), Poria Sclerotium (4.0 g, sclerotium of Poria cocos Wolf), Cnidium Rhizome (3.0 g, rhizome of Cnidium officinale Makino), Uncaria Hook (UH: 3.0 g, hook of Uncaria rhynchophylla Miquel), Japanese Angelica Root (3.0 g, root of Angelica acutiloba Kitagawa), Bupleurum Root (2.0 g, root of Bupleurum falcatum Linné), and Glycyrrhiza (1.5 g, root and stolon of Glycyrrhiza uralensis Fisher) supplied by Tsumura \& Co. (Tokyo, Japan). Each plant material was authenticated by identification of external morphology and marker compounds of plants specimens, according to the methods of Japanese Pharmacopoeia and our company's standard. Regarding components contained in yokukansan extract, 25 compounds including seven UH alkaloids have been identified by three-dimensional high-performance liquid chromatographic analysis [12].

Seven UH alkaloids [20], i.e., three indole alkaloids (hirsutine: HIT, hirsuteine: HTE, geissoschizine methyl ether: GM), and four oxindole alkaloids (rhynchophylline: RP, isorhynchophylline: IRP, corynoxeine: CX, and isocorynoxeine: ICX), used in the present study were supplied by the Botanical Raw Materials Research Department of Tsumura \& Co. (Ibaraki, Japan). 


\subsection{Reagents Used in Cell Culture Experiments}

Dispase I and DNase I were purchased from Roche Molecular Biochemicals (Mannheim, Germany). Dulbecco's modified Eagle's medium (DMEM), 4',6-diamidino-2-phenylindole dihydrochloride (DAPI), and phalloidinconjugated Alexa Fluor 488 were purchased from Life Technologies (Gaithersburg, MD, USA). Fetal bovine serum (FBS), trypsin, sodium pentobarbital, glucose, kanamycin, insulin, transferrin, bovine serum albumin (BSA), progesterone, putrescine, sodium selenite, thyroxine (T4), and tri-iodothyronine (T3) were purchased from Sigma-Aldrich (St. Louis, MO, USA). Monoclonal antibodies against A2B5 (clone MAB312R) and myelin basic protein (MBP, clone SKB3) were purchased from Merck Millipore (Billerica, MA, USA). A monoclonal antibody against O4 (clone MAB1326) was purchased from R\&D Systems (Minneapolis, MN, USA). Secondary antibodies, goat anti-mouse IgM-conjugated Alexa Fluor 488 and goat anti-mouse IgG1-conjugated Alexa Fluor 594 were purchased from Life Technologies. Other chemicals were purchased from commercial sources.

\subsection{Preparation of Oligodendrocyte Precursor Cells (OPCs)}

OPCs were purified from primary mixed cell cultures of embryonic mouse cerebral hemispheres according to the procedure described by Seiwa et al. [8]. In brief, a pregnant mouse (ICR, Japan SLC, Hamamatsu, Japan) was killed on the 17th day of pregnancy under deep anesthesia by diethyl ether, and the embryos were removed. Cerebral hemispheres from the embryos were washed with DMEM and enzymatically dissociated in a mixture of $0.3 \%$ dispase I and $0.05 \%$ DNase I in DMEM. The dissociated cells were sieved through $70-\mu \mathrm{m}$-pore-sized nylon mesh (\#2350) (Becton Dickinson, Franklin, NJ, USA) and then seeded on 10-cm diameter poly-L-lysine (Sigma-Aldrich)-coated culture dishes (Becton Dickinson) at a density of $1.3 \times 10^{7} /$ dish in DMEM containing $10 \%$ FBS. After $5 \mathrm{~d}$ culture, the cells were dissociated with $0.2 \%$ trypsin in phosphate-buffered saline and centrifuged for $10 \mathrm{~min}$ at $1000 \mathrm{rpm}$ and $4^{\circ} \mathrm{C}$. The cells were re-suspended in a serum-free medium consisting of DMEM supplemented with glucose $(5.6 \mathrm{mg} / \mathrm{ml})$, kanamycin $(60 \mathrm{mg} / \mathrm{ml})$, insulin $(5 \mu \mathrm{g} / \mathrm{ml})$, transferrin $(0.5$ $\mu \mathrm{g} / \mathrm{ml})$, BSA $(100 \mu \mathrm{g} / \mathrm{ml})$, progesterone $(0.06 \mathrm{ng} / \mathrm{ml})$, putrescine $(16 \mu \mathrm{g} / \mathrm{ml})$, sodium selenite $(40 \mathrm{ng} / \mathrm{ml})$, T4 (40 $\mathrm{ng} / \mathrm{ml})$, and T3 $(30 \mathrm{ng} / \mathrm{ml})$. The re-suspended cells $\left(3.0 \times 10^{6}\right.$ cells/ml) were incubated for $2 \mathrm{~h}$ at $37^{\circ} \mathrm{C}$ in $5 \%$ $\mathrm{CO} 2$. Neurons and astrocytes have been demonstrated to be eliminated from the mixed cell culture by this procedure. The purity of OPCs in the culture was examined by an immunocytochemical stain using A2B5, which is a specific marker of OPCs. We confirmed that $95 \%$ of cells were OPCs in preliminary examination. The purified OPCs were used in the following experiments to evaluate the effects of yokukansan and UH components on proliferation and differentiation of oligodendrocytes.

\subsection{Examination of Effects of Yokukansan and GM on Proliferation and Differentiation of Oligodendrocytes}

Purified OPCs $\left(5.5 \times 10^{4}\right.$ cells $\left./ \mathrm{cm}^{2}\right)$ were cultured in a chamber slide (Thermo Fisher Scientific, Waltham, MA, USA) for $2 \mathrm{~d}$ to evaluate proliferation or $4 \mathrm{~d}$ to evaluate differentiation in the serum-free medium (composition: see previous section) with yokukansan (final concentration: 100 or $200 \mu \mathrm{g} / \mathrm{ml}$ ), GM (final concentration: 1 or 3 $\mu \mathrm{M}$ ), or without either as a control. Yokukansan and GM were dissolved in $0.5 \%$ dimethyl sulfoxide (DMSO). The effect of yokukansan or GM on proliferation of OPCs was evaluated on the second day of the culture by counting A2B5-positive cells. The effect of yokukansan or GM on differentiation of oligodendrocyte was evaluated on the fourth day by counting O4-positive cells. Namely, the density (number/10 $\mathrm{mm}^{2}$ ) of A2B5-positive or O4-positive cell was calculated by counting six areas $\left(10 \mathrm{~mm}^{2} /\right.$ area $)$ in each sample. The density in each group was calculated as the mean \pm S.E.M. of six separate experiments, and the change of test substance was finally represented as a percentage of the number of cells in control group.

\subsection{Immunocytochemistry}

To determine the cellular specificity of the OPCs and oligodendrocytes, the cells were fixed with $4 \%$ paraformaldehyde in $0.1 \mathrm{M}$ phosphate buffer for $15 \mathrm{~min}$. The fixed cells were further treated with $0.1 \%$ Triton X-100PBS for $15 \mathrm{~min}$. Immunocytochemical stainings were performed according to the procedure previously reported [21]; monoclonal antibodies against gangliosides (A2B5, IgM, 1:100), sulfatide (O4, IgM, 1:100), and MBP (IgG1, 1:100) were used for oligodendrocyte lineage analysis. Goat anti-mouse IgM-conjugated Alexa Fluor 488 (1:200) and goat anti-mouse IgG1-conjugated Alexa Fluor 594 (1:200) were used as secondary antibodies. A 
mixture of secondary antibodies and phalloidin (30 units/ml) was used for staining of F-actin. Fluorescence and digital images were observed and recorded with a confocal laser scanning microscope FV-1000 (Olympus, Tokyo, Japan) or fluorescent microscope BZ-8100 (Keyence, Osaka, Japan).

\subsection{WST-8 Assay}

The WST-8 assay was performed according to the method reported by Ishiyama et al. [22]. Purified OPCs (1.5 $\times$ $10^{4} \mathrm{cells} / 100 \mu \mathrm{l} /$ well) were cultured in a non-coated 96-well plate (Becton Dickinson) for $48 \mathrm{~h}$ with or without test substances, which were dissolved in $0.5 \%$ dimethyl sulfoxide (DMSO). Then, $10 \mu$ of proliferation reagent WST-8 (Dojin-do, Kumamoto, Japan) was added to each well and incubated at $37^{\circ} \mathrm{C}$ for $1 \mathrm{~h}$. Absorbance of the WST-8 formazan was measured at $450 \mathrm{~nm}$ using a Infinite M200 plate reader (Tecan, Grödig, Austria).

\subsection{Statistical Analysis}

Data are presented as the mean \pm S.E.M. The statistical significance of differences between groups in cell culture experiments was assessed by Student's t-test or one-way analysis of variance (ANOVA) followed by Dunnett's post hoc test. The significance level in each statistical analysis was accepted at $p<0.05$.

\section{Results}

\subsection{Proliferation and Differentiation of Oligodendrocytes}

The proliferation and differentiation of processes in high-purity mouse cortical OPCs during culture in normal medium for four days were examined. As shown in Figure 1, proliferation of OPCs having no processes (Figure 1(a)) was observed mainly on the second day of culture. These OPCs were specifically identified by A2B5 antibody (Figure 1(a)). On the fourth day of culture, cells in the differentiation stage mainly having various numbers of

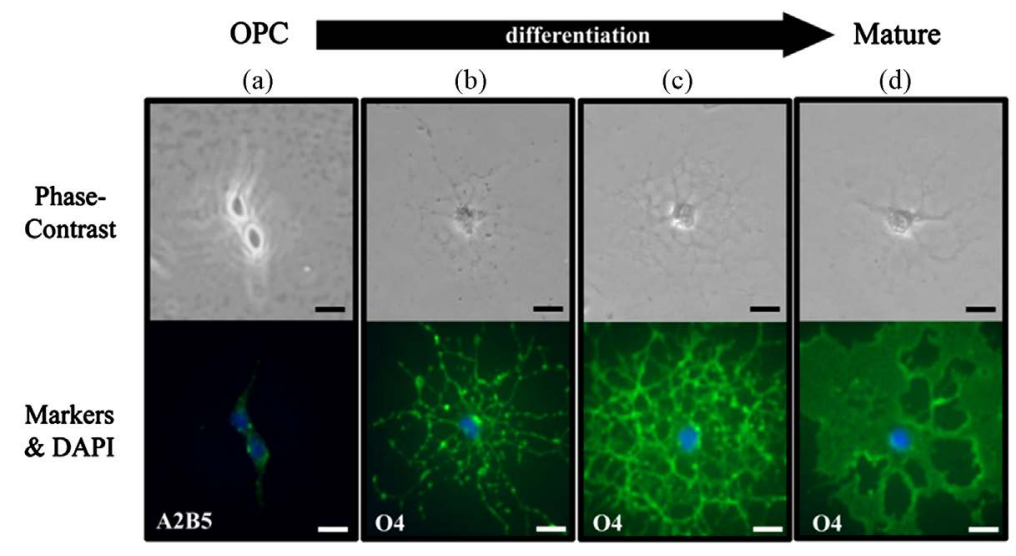

(e): Mature cell

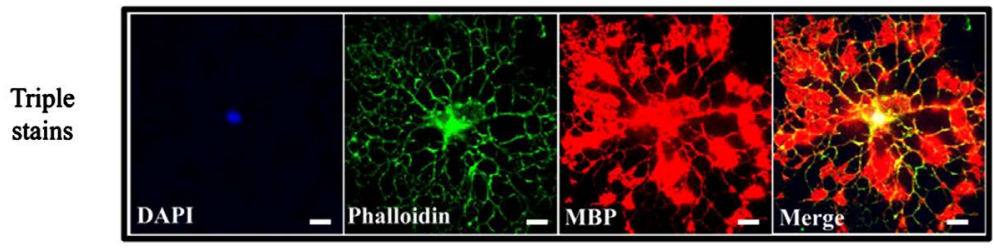

Figure 1. Proliferation of OPCs and differentiation to oligodendroctes. Proliferation of OPCs was evaluated on the second day of culture, and differentiation to oligodendrocytes was evaluated on the fourth day of culture. (a) shows OPC, and (b)-(d) show differentiated cells having various numbers of processes. An OPC was specifically identified as A2B5-positive (a). Cells in various stages of differentiation observed during differentiation process were identified as O4positive cells (b)-(d). A mature cell was identified as an MBP-positive cell (e). (e) shows a mature cell triple stained with DAPI (nucleus), phalloidin (F-actin), and MBP(myelin basic protein). Scale bars $=10 \mu \mathrm{m}$. 
processes were observed. For example, immature differentiated cells in the early stage had several processes (Figure 1(b)), and mature differentiated cells in the late stage had abundant processes (Figure 1(c) and Figure 1(d)). All stages of differentiated cells were specifically identified by using O4 antibody (Figures 1(b)-(d)). When mature cells morphologically characterized by the appearance of abundant processes, as shown in Figure 1(c) or Figure 1(d), were triple stained with DAPI (nucleus), phalloidin (F-actin), and MBP, the mature cell was identified as MBP-positive (Figure 1(e)), while A2B5-positive OPCs (Figure 1(a)) and early stage O4-positive immature differentiated cells (Figure 1(b)) were observed as MBP-negative cells (data not shown here). Furthermore, in a preliminary study to examine the experimental conditions, the death of mature cells was observed on the fifth day of culture (data not shown here). Therefore, in the present study, the effects of yokukansan on the proliferation and differentiation of oligodendrocytes were evaluated by counting the A2B5-positive cells on the second day and the O4-positive cells on the fourth day.

\subsection{Effects of Yokukansan on Proliferation and Differentiation of Oligodendrocytes}

OPCs were cultured for four days in the culture medium without (control) or with yokukansan (final concentration: 100 or $200 \mu \mathrm{g} / \mathrm{ml}$ ). The number of A2B5-positive cell in control, $100 \mu \mathrm{g} / \mathrm{ml}$ yokukansan, and $200 \mu \mathrm{g} / \mathrm{ml}$ yokukansan groups on the second day of culture were $123.5 \pm 7.7 \mathrm{cells} / 10 \mathrm{~mm}^{2}, 158.3 \pm 7.9$ cells $/ 10 \mathrm{~mm}^{2}$ and $165.5 \pm 9.9$ cells $/ 10 \mathrm{~mm}^{2}$, respectively. Figure 2 shows these values as a percentage of the number of cells in control group. A2B5-positive cell was significantly increased by treatment with yokukansan (100 and $200 \mu \mathrm{g} / \mathrm{ml})$ compared to that of the control.

The number of O4-positive cell in control, $100 \mu \mathrm{g} / \mathrm{ml}$ yokukansan, and $200 \mu \mathrm{g} / \mathrm{ml}$ yokukansan groups on the fourth day of culture were $8.3 \pm 1.2$ cells $/ 10 \mathrm{~mm}^{2}, 17.8 \pm 1.3$ cells $/ 10 \mathrm{~mm}^{2}$ and $17.2 \pm 0.75$ cells $/ 10 \mathrm{~mm}^{2}$, respectively. Figure 3 shows these values as a percentage of the number of cells in control group. Yokukansan (100 and $200 \mu \mathrm{g} / \mathrm{ml}$ ) significantly increased the number of O4-positive cell compared to that of the control.

\subsection{Screening Assay for Selection of Active Components in UH}

A WST-8 assay as a concise method of counting cells or proliferation was performed at a fixed concentration of $10 \mu \mathrm{M}$ to identify the active components of seven (HIT, HTE,GM, RP, IRP, CX, and ICX) of UH, which is one of the constituent galenicals of yokukansan. A significant proliferative effect was found only for GM (Figure 4(a)) and was observed with a concentration dependency (0.1, 1.0, and $10.0 \mu \mathrm{M}$ of GM) (Figure 4(b)).

\subsection{Effects of GM on Proliferation and Differentiation of Oligodendrocytes}

The proliferation and differentiation effects of GM, identified by the WST-8 screening assay, were evaluated by using the same assay as the case of yokukansan. GM $(1.0$, and 3.0 $\mu \mathrm{M})$ increased the number of A2B5-positive

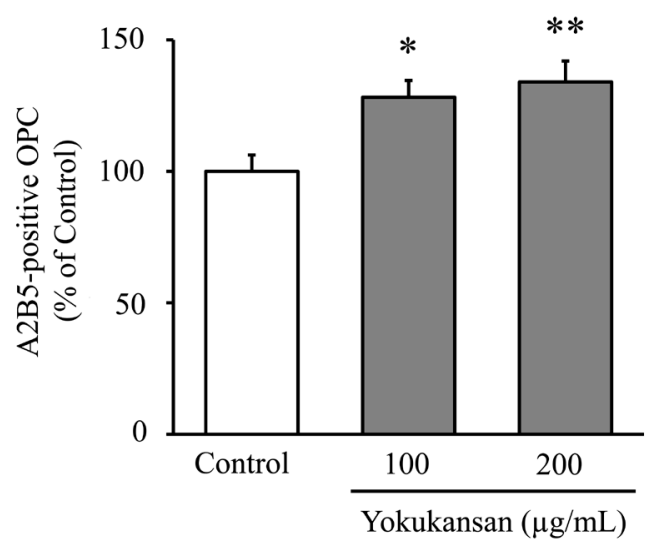

Figure 2. Effect of yokukansan on proliferation of OPC. OPCs were cultured for four days in the culture medium without (control) or with yokukansan (final concentration: 100 or 200 $\mu \mathrm{g} / \mathrm{ml}$ ). The number of A2B5-positive cells was counted on the second day. Each value calculated as a percentage of the control represents the mean \pm S.E.M. $(\mathrm{n}=6) .{ }^{*} p<0.05$ and ${ }^{* *} p$ $<0.01$ vs. control: one-way ANOVA + Dunnett's test. 


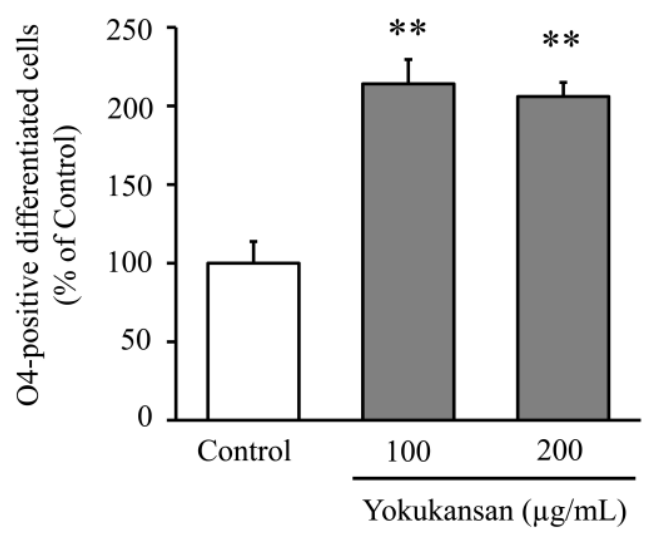

Figure 3. Effect of yokukansan on differentiation of oligodendrocytes. OPCs were cultured for four days in the culture medium without (control) or with yokukansan (final concentration: 100 or $200 \mu \mathrm{g} / \mathrm{ml}$ ). The number of O4-positive differentiated cells was counted on the fourth day. Each value calculated as a percentage of the control represents the mean \pm S.E.M. $(n=$ 6). ${ }^{* *} p<0.01$ vs. control: one-way ANOVA + Dunnett’s test.

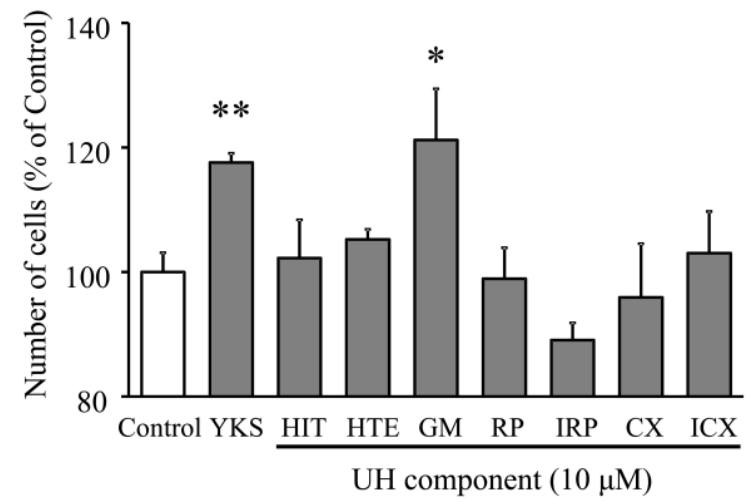

(a)

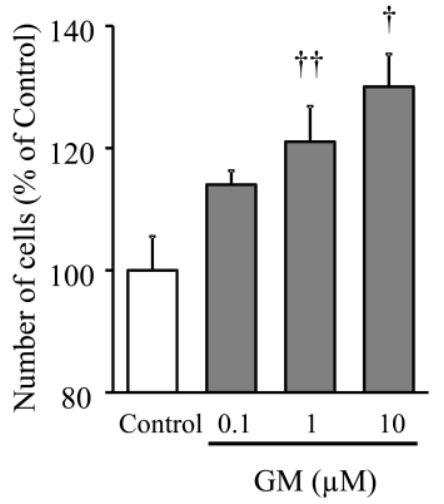

(b)

Figure 4. Screening of active components from UH alkaloids. A WST-8 assay was performed at a fixed concentration of $10 \mu \mathrm{M}$ on the second day of OPC culture (a). Concentration-dependency of GM identified by the screening assay was also evaluated by the same procedure. Each value calculated as a percentage of the control represents the mean \pm S.E.M. $(n=3)$. Effect of each test substance was compared to control using Student's t-test. ${ }^{*} p<0.05$ and ${ }^{* * *} p$ $<0.01$ vs. control. Concentration dependency was evaluated using one-way ANOVA + Dunnett's test. ${ }^{\dagger} p<0.05$ and ${ }^{\dagger+} p<0.01$ vs. control. Abbreviations: yokukansan (YKS), hirsutine (HIT), hirsuteine (HTE), geissoschizine methyl ether (GM), rhynchophylline (RP), isorhynchophylline (IRP), corynoxeine (CX), isocorynoxeine (ICX), and Uncaria Hook (UH).

cells on the second day (Figure 5) and O4-positive cells on the fourth day (Figure 6) compared to those of the corresponding controls.

\section{Discussion}

We previously demonstrated that yokukansan ameliorated not only the behavioral and psychological symptoms of thiamine-deficient rats but also the demyelination and degeneration of their oligodendrocytes [15] [16]. These findings led us to speculate that the effectiveness of yokukansan may be related to the function or repair of oligodendrocytes, which are responsible for formation of the myelin sheath. In the present study, therefore, to clarify whether or not yokukansan affects oligodendrocytes, its effects on proliferation and differentiation of oligodendrocytes were examined using high-purity mouse cortical OPCs.

We demonstrated in this study that OPC became mature oligodendrocytes by proliferation and differentiation in four days. The number of cells positive to A2B5, which is a marker of OPCs, was significantly increased by 


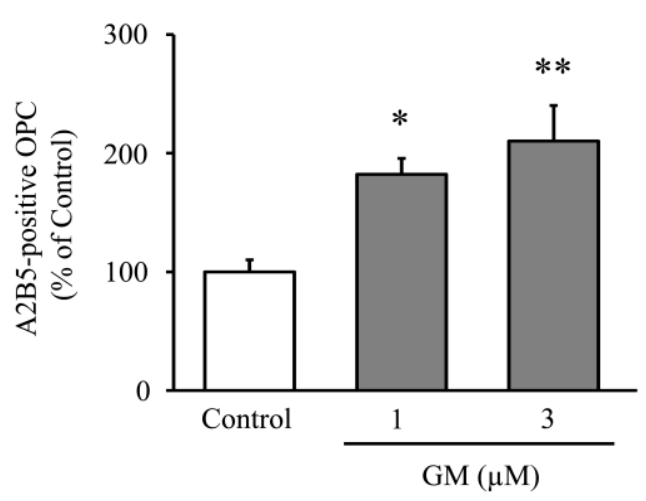

Figure 5. Effect of GM on proliferation of OPCs. OPCs were cultured for four days in the culture medium without (control) or with GM (final concentration: 1 or $3 \mu \mathrm{M}$ ). The number of A2B5-positive cells was counted on the second day. Each value calculated as a percentage of the control represents the mean \pm S.E.M. $(\mathrm{n}=6) .{ }^{*} p<0.05$ and ${ }^{* *} p<0.01$ vs. control: one-way ANOVA + Dunnett's test.

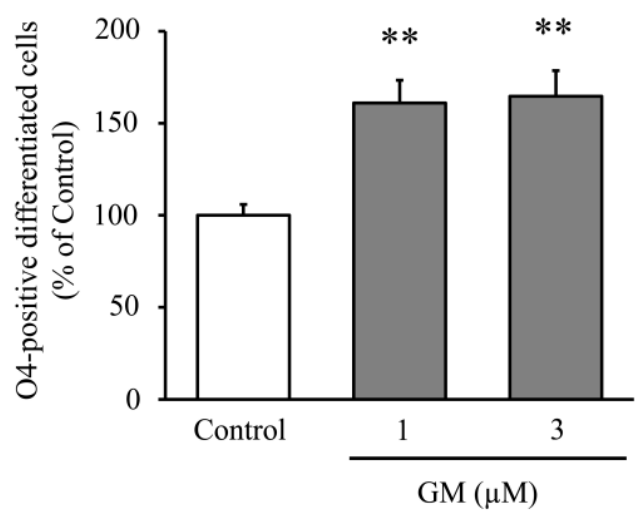

Figure 6. Effect of GM on differentiation of oligodendrocytes. OPCs were cultured for four days in the culture medium without (control) or with GM (final concentration: 1 or $3 \mu \mathrm{M}$ ). The number of O4-positive differentiated cells was counted on the fourth day. Each value calculated as a percentage of the control represents the mean \pm S.E.M. $(\mathrm{n}=6) .{ }^{* *} p<0.01$ vs. control: oneway ANOVA + Dunnett's test.

yokukansan on the second day of culture, and the number of cells positive to O4, which is a marker of various staged differential cells, was significantly increased by yokukansan on the fourth day of culture, suggesting that yokukansan facilitates not only the proliferation of OPCs but also their differentiation to oligodendrocytes.

Oligodendrocyte development (proliferation and differentiation) and protection are controlled by a variety of extracellular growth and differentiation factors including extracellular signal regulated kinase 1/2 (ERK 1/2) and phosphatidylinositol 3-kinase (PI3K)/Akt [23]-[25] like those in neuronal cells [26]-[28]. More recently, Kubota et al. demonstrated that yokukansan activated ERK 1/2 and PI3K/Akt in PC12 cells [29]. Yokukansan was also demonstrated to possess neuroprotective and neurogenerative effects in vivo and in vitro [19] [30] [31]. In addition to these findings, $\mathrm{UH}$, a constituent galenical of yokukansan, has been reported to possess neuroprotective effects in vivo and in vitro [19] [30] [32]-[35]. UH was also reported as a candidate for the pharmacological effects of yokukansan such as amelioration of aggressiveness [18]. From these findings, we inferred a possibility that UH might contain active components related to the development of oligodendrocytes.

A screening assay by WST-8 identified GM from seven kinds of alkaloid components of UH as a candidate for the active component (Figure 4). Indeed, GM increased the number of A2B5-positive cells on the second day (Figure 5) and O4-positive cells on the fourth day (Figure 6) as yokukansan did (Figure 2 and Figure 3). These results suggest that GM promotes the proliferation and differentiation of oligodendrocytes. Recently, Mo- 
rita et al. examined the effect of GM on oligodendrocyte by double immunolabeling using BrdU-labeled NG2 and GSTpi for oligodendrocyte lineage in cuprizone (CPZ)-fed mice, an animal model of schizophrenia [36]. GM treatment significantly increased BrdU-positive GSTpi+ cells, but had no significant effect on BrdU-positive NG2+ cells. From these results, they suggested that GM had specific differentiation-promoting effect on oligodendrocyte progenitor cells, and likely acts as a remyelination promoter in the medial prefrontal cortex of CPZ mice. This in vivo evidence also partly supports our present result that GM is an active component responsible for the proliferation and differentiation effects of yokukansan on oligodendrocytes. We previously demonstrated that GM in yokukansan administered orally was absorbed into the blood and crosses the blood brain barrier [37], suggesting that GM included in yokukansan reaches the brain. These results, taken together, further suggest a possibility that GM may promote the OPC lineage progression and function as remyelination in the brain. Unfortunately, the mechanism of the OPC lineage progression by GM has not been clarified so far. As possible mechanism, we are inferring involvement of extracellular signal regulated kinase pathway or protein kinase C (PKC), because yokukansan has been reported to activate ERK 1/2 and PI3K/Akt [29] as already described, or to inhibit PKC activity [38]. OPC differentiation has been reported to be promoted by inhibition of PKC [3] or to be inhibited by activation of PKC [39]. The detailed examination to clarify the molecular mechanism will be necessary in the future.

\section{Conclusion}

In conclusion, our results suggest a possibility that yokukansan promotes the proliferation and differentiation of oligodendrocytes, and further that the GM contained in UH is one of the active components responsible for the effect of yokukansan.

\section{References}

[1] Keirstead, H.S. and Blakemore, W.F. (1999) The Role of Oligodendrocytes and Oligodendrocyte Progenitors in CNS Remyelination. Advances in Experimental Medicine and Biology, 468, 183-197. http://dx.doi.org/10.1007/978-1-4615-4685-6_15

[2] Morrell, P. and Quarles, R.H. (1999) Myelin Formation, Structure and Biochemistry. In: Siegel, G.J., Agranoff, B.W., Alber, R.W., Risher, S.K. and Uhler, M.D., Eds., Basic Neurochemistry, 6th Edition, Raven Press, New York, 70-93.

[3] Baer, A.S., Syed, Y.A., Kang, S.U., Mitteregger, D., Vig, R., Ffrench-Constant, C., Franklin, B.J.M., Altmann, F., Lubec, G. and Kotter, M.R. (2009) Myelin-Mediated Inhibition of Oligodendrocyte Precursor Differentiation Can Be Overcome by Pharmacological Modulation of Fyn-RhoA and Protein Kinase C Signaling. Brain, 132, 465-481. http://dx.doi.org/10.1093/brain/awn334

[4] Mi, S., Miller, R.H., Tang, W., Lee, X., Hu, B., Wu, W., Zhang, Y., Shields, C.B., Zhang, Y., Miklasz, S., Shea, D., Mason, J., Franklin, R.J.M., Ji, B., Jung, V. and Pepinsky, B. (2009) Promotion of Clinical Nervous System Remyelination by Induced Differentiation of Oligodendrocyte Precursor Cells. Annals of Neurology, 65, 304-315. http://dx.doi.org/10.1002/ana.21581

[5] Kobayashi, J., Seiwa, C., Sakai, T., Gotoh, M., Komatsu, Y., Yamamoto, M., Fukutake, M., Matsuno, K., Sakurai, Y., Kawano, Y. and Asou, H. (2003) Effect of a Traditional Chinese Herbal Medicine, Ren-Shen-Yang-Rong-Tang (Japanese Name: Ninjin-Youei-To), on Oligodendrocyte Precursor Cells from Aged-Rat Brain. International Immunopharmacology, 3, 1027-1039. http://dx.doi.org/10.1016/S1567-5769(03)00101-2

[6] Akundi, R.S. and Rivkees, S.A. (2009) Hypoxia Alters Cell Cycle Regulatory Protein Expression and Induces Premature Maturation of Oligodendrocyte Precursor Cells. PLoS ONE, 4, Article ID: e4739. http://dx.doi.org/10.1371/journal.pone.0004739

[7] Hazell, A.S., Todd, K.G. and Butterworth, R.F. (1998) Mechanism of Neuronal Cell Death in Wernicke’s Encephalopathy. Metabolic Brain Disease, 13, 97-122. http://dx.doi.org/10.1023/A:1020657129593

[8] Seiwa, C., Kojima-Akikawa, K., Matsumoto, I. and Asou, H. (2002) CNS Myelinogenesis in Vitro: Myelin Basic Protein Deficient Shiverer Oligodendrocytes. Journal of Neuroscience Research, 69, 305-317. http://dx.doi.org/10.1002/jnr.10291

[9] Seiwa, C., Yamamoto, M., Tanaka, K., Fukutake, M., Ueki, T., Takeda, S., Sakai, R., Ishige, A., Watanabe, K., Akita, M., Yagi, T., Tanaka, K. and Asou, H. (2007) Restoration of FcR $\gamma /$ Fyn Signaling Repairs Central Nervous System Demyelination. Journal of Neuroscience Research, 85, 954-966. http://dx.doi.org/10.1002/jnr.21196

[10] Iwasaki, K., Satoh-Nakagawa, T., Maruyama, M., Monma, Y., Nemoto, M., Tomita, N., Tanji, H., Fujiwara, H., Seki, T., Fujii, M., Arai, H. and Sasaki, H. (2005) A Randomized, Observer-Blind, Controlled Trial of the Traditional Chi- 
nese Medicine Yi-Gan San for Improvement of Behavioral and Psychological Symptoms and Activities of Daily Living in Dementia Patients. Journal of Clinical Psychiatry, 66, 248-252. http://dx.doi.org/10.4088/JCP.v66n0214

[11] Iwasaki, K., Kosaka, K., Mori, H., Okitsu, R., Furukawa, K., Manabe, Y., Yoshita, M., Kanamori, A., Ito, N., Wada, K., Kitayama, M., Horiguchi, J., Yamaguchi, S., Katayama, S., Fukuhara, R., Ouma, S., Nakano, S., Hashimoto, M. and Kinoshita, T. (2012) Improvement in Delusions and Halucinations in Patients with Dementia with Lewy Bodies upon Administration of Yokykansan, a Traditional Japanese Medicine. Psychogeriatrics, 12, 235-241. http://dx.doi.org/10.1111/j.1479-8301.2012.00413.x

[12] Mizukami, K., Asada, T., Kinoshita, T., Tanaka, K., Sonohara, K., Nakai, R., Yamaguchi, K., Hanyu, H., Kanaya, K., Takao, T., Okada, M., Kudo, S., Kotoku, H., Iwakiri, M., Kurita, H., Miyamura, T., Kawasaki, Y., Omori, K., Shiozaki, K., Odawara, T., Suzuki, T., Yamada, S., Nakamura, Y. and Toba, K. (2009) A Randomized Cross-Over Study of a Traditional Japanese Medicine (Kampo), Yokukansan, in the Treatment of the Behavioural and Psychological Symptoms of Dementia. The International Journal of Neuropsychopharmacology, 12, 191-199. http://dx.doi.org/10.1017/S146114570800970X

[13] Nagata, K., Yokoyama, E., Yamazaki, T., Takano, D., Maeda, T., Takahashi, S. and Terayama, Y. (2012) Effects of Yokukansan on Behavioral and Psychological Symptoms of Vascular Dementia: Open-Label Trial. Phytomedicine, 19, 524-528. http://dx.doi.org/10.1016/j.phymed.2012.02.008

[14] Matsuda, Y., Kishi, T., Shibayama, H. and Iwata, N. (2013) Yokukansan in the Treatment of Behavioral and Psychological Symptoms of Dementia: A Systematic Review and Meta-Analysis of Randomized Controlled Trials. Human Psychopharmacology: Clinical and Experimental, 28, 80-86. http://dx.doi.org/10.1002/hup.2286

[15] Ikarashi, Y., Iizuka, S., Imamura, S., Yamaguchi, T., Sekiguchi, K., Kanno, H., Kawakami, Z., Yuzurihara, M., Kase, Y. and Takeda, S. (2009) Effects of Yokukansan, a Traditional Japanese Medicine, on Memory Disturbance and Behavioral and Psychological Symptoms of Dementia in Thiamine-Deficient Rats. Biological \& Pharmaceutical Bulletin, 32, 1701-1709. http://dx.doi.org/10.1248/bpb.32.1701

[16] Iizuka, S., Kawakami, Z., Imamura, S., Yamaguchi, T., Sekiguchi, K., Kanno, H., Ueki, T., Kase, Y. and Ikarashi, Y. (2010) Electron-Microscopic Examination of Effects of Yokukansan, a Traditional Japanese Medicine, on Degeneration of Cerebral Cells in Thiamine-Deficient Rats. Neuropathology, 30, 524-536.

http://onlinelibrary.wiley.com/doi/10.1111/j.1440-1789.2010.01101.x/full

[17] Fujiwara, H., Takayama, S., Iwasaki, K., Tabuchi, M., Yamaguchi, T., Sekiguchi, K., Ikarashi, Y., Kudo, Y., Kase, Y., Arai, H. and Yaegashi, N. (2011) Yokukansan, a Traditional Japanese Medicine, Ameliorates Memory Disturbance and Abnormal Social Interaction with Anti-Aggregation Effect of Cerebral Amyloid $\beta$ Proteins in Amyloid Precursor Protein Transgenic Mice. Neuroscience, 180, 305-313. http://dx.doi.org/10.1016/j.neuroscience.2011.01.064

[18] Nishi, A., Yamaguchi, T., Sekiguchi, K., Imamura, S., Tabuchi, M., Kanno, H., Nakai, Y., Hashimoto, K., Ikarashi, Y. and Kase, Y. (2012) Geissoschizine Methyl Ether, an Alkaloid in Uncaria Hook, Is a Potent Serotonin 1A $_{\text {Receptor }}$ Agonist and Candidate for Amelioration of Aggressiveness and Sociality by Yokukansan. Neuroscience, 201, 124-136. http://dx.doi.org/10.1016/j.neuroscience.2012.01.037

[19] Kawakami, Z., Kanno, H., Ikarashi, Y. and Kase, Y. (2011) Yokukansan, a Kampo Medicine, Protects against Glutamate Cytotoxicity Due to Oxidative Stress in PC12 Cells. Journal of Ethnopharmacology, 134, 74-81. http://dx.doi.org/10.1016/j.jep.2010.11.063

[20] Yuzurihara, M., Ikarashi, Y., Goto, K., Sakakibara, I., Hayakawa, T. and Sasaki, H. (2002) Geissoschizine Methyl Ether, an Indole Alkaloid Extracted from Uncariae Ramulus et Uncus, Is a Potent Vasorelaxant of Isolated Rat Aorta. European Journal of Pharmacology, 444, 183-189. http://dx.doi.org/10.1016/S0014-2999(02)01623-0

[21] Ueki, T., Tsuruo, Y., Yamamoto, Y., Yoshimura, K., Takanaga, H., Seiwa, C., Motojima, K., Asou, H. and Yamamoto, M. (2012) A New Monoclonal Antibody, 4F2, Specific for the Oligodendroglial Cell Lineage, Recognizes ATP-Dependent RNA Helicase Ddx54: Possible Association with Myelin Basic Protein. Journal of Neuroscience Research, 90, 48-59. http://dx.doi.org/10.1002/jnr.22736

[22] Ishiyama, M., Miyazono, Y., Sasamoto, K., Ohkura, Y. and Ueno, K. (1997) A Highly Water-Soluble Disulfonated Tetrazolium Salt as a Chromogenic Indicator for NADH as Well as Cell Viability. Talanta, 44, 1299-1305. http://dx.doi.org/10.1016/S0039-9140(97)00017-9

[23] Arai, K. and Lo, E.H. (2010) Astrocytes Protect Oligodendrocyte Precursor Cell via MEK/ERK and PI3K/Akt Signaling. Journal of Neuroscience Research, 88, 758-763. http://www.ncbi.nlm.nih.gov/pmc/articles/PMC3705578/

[24] Bibollet-Bahena, O. and Almazan, G. (2009) IGF-1-Stimulated Protein Synthesis in Oligodendrocyte Progenitors Requires PI3K/mTOR/Akt and MEK/ERK Pathways. Journal of Neurochemistry, 109, 1440-1451. http://dx.doi.org/10.1111/j.1471-4159.2009.06071.x

[25] Fyffe-Maricich Karlo, J.C., Landreth, G.E. and Miller, R.H. (2011) The ERK2 Mitogen-Activated Protein Kinase Regulates the Timing of Oligodendrocyte Differentiation. The Journal of Neuroscience, 31, 843-850. http://dx.doi.org/10.1523/JNEUROSCI.3239-10.2011 
[26] Vaudry, D., Stork, P.J., Lazarovici, P. and Eiden, L.E. (2002) Signaling Pathways for PC12 Cell Differentiation: Making the Right Connections. Science, 296, 1648-1649. http://dx.doi.org/10.1126/science.1071552

[27] Hetman, M. and Gozdz, A. (2004) Role of Extracellular Signal Regulated Kinases 1 and 2 in Neuronal Survival. European Journal of Biochemistry, 271, 2050-2055. http://dx.doi.org/10.1111/j.1432-1033.2004.04133.x

[28] Chen, J.Y., Lin, J.R., Cimprich, K.A. and Meyer, T. (2012) Two-Dimensional ERK-AKT Signaling Code for an NGFTriggered Cell-Fate Decision. Molecular Cell, 45, 196-209. http://dx.doi.org/10.1016/j.molcel.2011.11.023

[29] Kubota, K., Sano, K., Shiraishi, A., Beppu, N., Nogami, A., Uchida, N., Takasaki, K., Katsurabayashi, S., Mishima, K., Nishimura, R., Fujiwara, M. and Iwasaki, K. (2013) Yokukansan, a Traditional Japanese Herbal Medicine, Promotes Neurite Outgrowth in PC12 Cells through the Activation of Extracellular Signal Regulated Kinase 1/2 and Phosphatidylinositol 3-Kinase/Akt. Journal of Traditional Medicines, 30, 102-113.

[30] Kawakami, Z., Kanno, H., Ikarashi, Y. and Kase, Y. (2011) Isoliquiritigenin Is a Novel NMDA Receptor Antagonist in Kampo Medicine Yokukansan. Cellular and Molecular Neurobiology, 31, 1203-1212. http://dx.doi.org/10.1007/s10571-011-9722-1

[31] Tanaka, Y. and Mizoguchi, K. (2009) Influence of Aging on Chondroitin Sulfate Proteoglycan Expression and Neural Stem/Progenitor Cells in Rat Brain and Improving Effects of a Herbal Medicine, Yokukansan. Neuroscience, 164, 1224-1234. http://dx.doi.org/10.1016/j.neuroscience.2009.08.060

[32] Shimada, Y., Goto, H., Ito, T., Sakakibara, I., Kubo, M., Sasaki, S. and Terasawa, K. (1999) Evaluation of the Protective Effects of Alkaloids Isolated from the Hooks and Stems of Uncaria sinensis on Glutamate-Induced Neuronal Death in Cultured Cerebellar Granule Cells from Rats. Journal of Pharmacology and Pharmacotherapeutics, 51, 715722. http://dx.doi.org/10.1211/0022357991772853

[33] Suk, K., Kim, S.Y., Leem, K., Kim, Y.O., Parl, S.Y., Hur, J., Beak, J., Lee, K.J., Zheng, H.Z. and Kim, H. (2002) Neuroprotection by Methanol Extract of Uncaria rhynchophylla against Global Cerebral Ischemia in Rats. Life Sciences, 70, 2467-2480. http://dx.doi.org/10.1016/S0024-3205(02)01534-5

[34] Lee, J., Son, D., Lee, P., Kim, S.-T., Kim, H., Kim, C.-J. and Lim, E. (2003) Alkaloid Fraction of Uncaria rhynchophylla Protects against N-Methyl-D-Aspartate-Induced Apoptosis in Rat Hippocampal Slices. Neuroscience Letters, 348, 51-55. http://dx.doi.org/10.1016/S0304-3940(03)00613-X

[35] Shim, J.S., Kim, H.G., Ju, M.S., Choi, J.G., Jeong, S.Y. and Oh, M.S. (2009) Effect of the Hook of Uncaria rhynchophylla on Neurotoxicity in the 6-Hydroxydopamine Model of Parkinson's Disease. Journal of Ethnopharmacology, 126, 361-365. http://dx.doi.org/10.1016/j.jep.2009.08.023

[36] Morita, S., Tatsumi, K., Makinodan, M., Okuda, H., Kishimoto, T. and Wanaka, A. (2014) Geissoschizine Methyl Ether, an Alkaloid from the Uncaria Hook Improves Remyelination after Cuprizone-Induced Demyelination in Medial Prefrontal Cortex of Adult Mice. Neurochemical Research, 39, 59-67. http://dx.doi.org/10.1007/s11064-013-1190-1

[37] Imamura, S., Tabuchi, M., Kushida, H., Nishi, A., Kanno, H., Yamaguchi, T., Sekiguchi, K., Ikarashi, Y. and Kase, Y. (2011) The Blood-Brain Barrier Permeability of Geissoschizine Methyl Ether in Uncaria Hook, a Galenical Constituent of the Traditional Japanese Medicine Yokukansan. Cellular and Molecular Neurobiology, 31, 787-793. http://dx.doi.org/10.1007/s10571-011-9676-3

[38] Kawakami, Z., Ikarashi, Y. and Kase, Y. (2010) Glycyrrhizin and Its Metabolite 18 Beta-Glycyrrhetinic Acid in Glycyrrhiza, a Constituent Herb of Yokukansan, Ameliorate Thiamine Deficiency-Induced Dysfunction of Glutamate Transport in Cultured Rat Cortical Astrocytes. European Journal of Pharmacology, 626, 154-158. http://dx.doi.org/10.1016/j.ejphar.2009.09.046

[39] Baron, W., de Jonge, J.C., de Vries, H. and Hoekstra, D. (1998) Regulation of Oligodendrocyte Differentiation: Protein Kinase C Activation Prevents Differentiation of O2A Progenitor Cells toward Oligodendrocytes. Glia, 22, 121-129. http://dx.doi.org/10.1002/(SICI)1098-1136(199802)22:2<121::AID-GLIA3>3.0.CO;2-A 


\section{Abbreviation}

OPC: oligodendrocyte precursor cell

UH: Uncaria hook

GM: geissoschizine methyl ether

CNS: central nervous system

BPSD: behavioral and psychological symptoms of dementia

TD: thiamine-deficient

HIT: hirsutine

HTE: hirsuteine

RP: rhynchophylline

IRP: isorhynchophylline

CX: corynoxeine

ICX: isocorynoxeine

DMEM: Dulbecco's modified Eagle’s medium

DAPI: 4',6-diamidino-2-phenylindole dihydrochloride

FBS: fetal bovine serum

BSA: bovine serum albumin

MBP: myelin basic protein

ERK: extracellular signal regulated kinase

PI3K: phosphatidylinositol 3-kinase

CPZ: cuprizone

BrdU: bromodeoxyuridine 